\title{
Silent Myocardial Infarction and Long-Term Risk of Frailty: The Atherosclerosis Risk in Communities Study
}

Fanghui $\mathrm{Li}^{1}{ }^{\mathrm{l}}, *$ Dongze $\mathrm{Li},{ }^{\mathrm{I}, *}$ Jing $\mathrm{Yu}^{2}{ }^{2} \mathrm{Yu}$ Jia,' Yi Liu, Yanmei Liu, ${ }^{3}$ Qinqin Wu, ${ }^{4}$ Xiaoyang Liao, ${ }^{5}$ Zhi Zeng,' Zhi Wan,' Rui Zeng ${ }^{1,6}$

'Department of Emergency Medicine and National Clinical Research Center for Geriatrics, Research Laboratory of Emergency Medicine, Disaster Medicine Center, West China Hospital, Sichuan University, Chengdu, People's Republic of China; ${ }^{2}$ West China School of Nursing, West China Hospital, Sichuan University, Chengdu, People's Republic of China; ${ }^{3}$ Chinese Evidence-Based Medicine Center, West China Hospital, Sichuan University, Chengdu, People's Republic of China; ${ }^{4}$ Health Management Center, West China Hospital, Sichuan University, Chengdu, People's Republic of China; ${ }^{5}$ Department of General Practice and National Clinical Research Center for Geriatrics, West China Hospital, Sichuan University, Chengdu, People's Republic of China; ' $D$ Department of Cardiology, West China Hospital, Sichuan University, Chengdu, People's Republic of China

*These authors contributed equally to this work

Correspondence: Rui Zeng; Zhi Wan Department of Cardiology, West China Hospital, Sichuan University, 37 Guoxue Road, Chengdu, 6I004I, Sichuan, People's Republic of China

$\mathrm{Tel}+86-28-8542246 \mathrm{I} ;+86-28-85421036$

Fax +86-28-85422288

Email zengrui_0524@।26.com;

303680215@qq.com
Background: Silent myocardial infarction (SMI) accounts for more than half of all MIs, and common risk factors and pathophysiological pathways coexist between SMI and frailty. The risk of frailty among patients with SMI is not well established. This study aimed to examine the association between SMI and frailty.

Methods and Results: This analysis included data from the Atherosclerosis Risk in Communities study. Patients without MI at baseline were eligible for inclusion. SMI was defined as electrocardiographic evidence of MI without clinical MI (CMI) after the baseline and until the fourth visit. Frailty was assessed during the fifth visit. A total of 4953 participants were included with an average age of $52.2 \pm 5.1$ years. Among these participants, $2.7 \%(n=135)$ developed SMI, and 2.9\% $(n=146)$ developed CMI. After a median follow-up time of $14.7(14.0-15.3)$ years, $6.7 \%(n=336)$ of the participants developed frailty. Patients with SMI and CMI were significantly more likely to become frail than those without MI (15.6\% vs $6.2 \%, P<0.001$ and $16.4 \%$ vs $6.2 \%, P<0.001$, respectively). After adjusting for confounders, SMI and CMI were found to be independent predictors of frailty (odds ratio $[\mathrm{OR}]=2.243,95 \%$ confidence interval $[\mathrm{CI}]=1.307-3.850, P=0.003$ and $\mathrm{OR}=2.164,95 \%$ $\mathrm{CI}=1.259-3.721, P=0.005$, respectively). The association was consistent among the subgroups of age, sex, race, diabetes, and hypertension.

Conclusion: In conclusion, both SMI and CMI were found to be associated with a higher risk of frailty. Future studies are needed to confirm the beneficial effects of screening for SMI as well as to implement standardized preventive treatment to reduce the risk of frailty.

Clinical Trial Registration: URL: https://www.clinicaltrials.gov; Unique identifier: NCT00005131.

Keywords: cardiovascular disease, silent myocardial infarction, frailty, electrocardiogram

\section{Introduction}

Frailty is a complex clinical syndrome that is defined as an increased vulnerability to stressors resulting from multiple impairments across different systems. It partly accounts for the heterogeneity between biological and chronological age. ${ }^{1}$ Generally, the prevalence of frailty increases with increasing age, and its incidence in community-dwelling older adults ( $>65$ years of age) ranges from $10 \%$ to $30 \%{ }^{1-3}$ Moreover, frailty is an independent predictor of falls, worsening mobility, disability, hospitalization, and death, subsequently becoming a public issue worldwide. ${ }^{4}$

Previous studies have demonstrated that cardiovascular disease and frailty are common and often coexist among older adults, ${ }^{5}$ risk factors and pathophysiological 
pathways may be shared between these two entities. A structured review found that nearly a third of older adults presenting to the hospital with acute coronary syndrome (ACS) are frail or pre-frail. ${ }^{6}$ In addition, studies have found that frailty is a crucial prognostic determinant among older adult patients with myocardial infarction (MI). ${ }^{1,7-10}$ Studies have shown an upward trend of frailty from $5.0 \%$ to $37 \%$ after $10-13$ years of clinical MI (CMI), and individuals with frailty are 2-5 times more likely to die or have major adverse cardiovascular events. ${ }^{11}$

Previous studies mostly focused on CMI, while silent MI (SMI) was paid less attention on due to lack characteristic chest pain symptoms. ${ }^{12,13}$ However, SMI constitutes up to $54 \%$ of all MIs, and more than $60 \%$ of all MIs in older adults ( $>60$ years of age). ${ }^{14-16}$ The prevalence of SMI ranges from $0.5 \%$ to $8.0 \%$ in the general population, rising to $27 \%$ in patients with suspected coronary artery disease (CAD). ${ }^{15,17,18}$ Thus, SMI is also a concern that need to be placed emphasis on. The risk of sudden cardiac death (SCD), heart failure (HF), and other adverse outcomes have been observed in both SMI and CMI, but have been worse among patients with SMI. ${ }^{19}$ With this in mind, SMI patients with frail may have worse outcomes. Additionally, SMI can be easily diagnosed by electrocardiogram (ECG). Thus, early identification of SMI and timely intervention may benefit in preventing and treating frailty.

However, there are limited data regarding the prevalence of SMI among individuals who later developed frailty in the general population, and the relationship between SMI and onset of frailty is not well established so far. Therefore, this study aimed to determine the prevalence of SMI and long-term frailty among the general population for individuals without any history of CAD. Moreover, this study compared the associations of SMI and CMI with frailty and examined the consistency of these associations in subgroups stratified by sex, race, and HF risk factors.

\section{Materials and Methods}

\section{Study Population}

Atherosclerosis Risk in Communities (ARIC) is a large, long-term prospective study involving participants between the ages of 45 and 64 years in four communities in the United States. The baseline survey was conducted from 1987 to 1989, the second, third, fourth, and fifth follow-ups was conducted from 1990 to 1992,1993 to
1995, 1996 to 1998, and 2011 to 2013, respectively. During the follow-up period, trained professionals assessed the participants' demographic characteristics, medical history, lifestyle, and hospitalization events through a standardized program to determine the participants' health status and events. The experimental protocol was established according to the ethical guidelines of the Helsinki Declaration. Each study site was approved by the institutional review boards at the collaborating medical centers: University of Mississippi Medical Center Institutional Review Board (Jackson Field Center); Wake Forest University Health Sciences Institutional Review Board (Forsyth County Field Center); University of Minnesota Institutional Review Board (Minnesota Field Center); and the Johns Hopkins School of Public Health Institutional Review Board (Washington County Field Center). And all participants provided a written informed consent during each follow-up. Data and materials can be obtained from the website: https://biolincc.nhlbi.nih.gov/ home/.

During the fifth follow-up, 6538 participants were assessed for frailty for the first time. Therefore, this analysis used the fifth follow-up population as the research outcome and the data from the first survey as the baseline. Some participants were excluded due to missing or poor quality ECG (671 participants), history of MI according to self-reports or ECG at the first visit (97 participants), history MI at the fifth visit (221 participants), lack of frailty assessment (398 participants), and lack of key covariates (17 participants).

\section{Frailty Phenotype}

All participants were assessed for frailty in the fifth visit, based on the definition of frailty phenotype previously operationalized in the ARIC study. ${ }^{20,21}$ This definition consists of five parts as follows: shrinking, weakness, slowness, poor energy, and low physical activity level. Shrinking is defined as weight loss and refers to the unconscious loss of $5 \%$ of total body weight or 10 pounds compared to the previous year. Weakness was assessed using a hydraulic grip dynamometer adjusted for sex and body mass index (BMI). Slowness was assessed by the time needed to walk for a distance of 15 feet based on the lowest quartile for sex and height. Poor energy was defined through the participants' answers to two statements ("I felt everything I did was an effort" and "I could not get going") based on the Center for Epidemiological Study's-Depression scale. Baecke 
physical activity questionnaire ${ }^{22}$ was used to classify low physical activity levels according to the lowest quartile for sex. Participants were classified as "frail" if they met three or more criteria, "pre-frail" if they meet one or two criteria, and "robust" if they did not meet any criteria.

\section{Silent and Clinical Myocardial Infarction}

During follow-up, trained staff used a MACPC (Marquette Electronics Inc, Milwaukee, WI) ECG to obtain a supine 12-lead ECG for each participant. All ECG results were read by two physicians who had received training in studying ECG. In the event of a discrepancy between the results by the two physicians, a senior practitioner made the decision. The presence of MI was defined by the Minnesota Code (MC) ECG classification as a minor Q/ QS wave abnormality (MC 1.3) plus a major ST-T abnormality (MC 4.1, MC 4.2, MC 5.1, or MC 5.2) or new appearance of a major $\mathrm{Q} / \mathrm{QS}$ wave abnormality (MC 1.1 or MC 1.2). CMI was determined by physicians based on chest pain, ECG evidence, history of coronary heart disease (CHD), and other relevant information. SMI was defined as a new MI, based on ECG evidence during the follow-up visit, without the presence of CMI at baseline. CMI and SMI were assessed after the baseline and until the fourth visit.

\section{Baseline Covariates}

At baseline (first visit), age, sex, race, alcohol consumption, smoking, and education levels were self-reported. The BMI was calculated by dividing weight in kilograms by height in meters squared. Blood samples were collected after fasting for 12 hours and tested in the central laboratory. The physical activity score is a score of sport index during leisure time based on the Baecke Questionnaire. ${ }^{23}$ Hypertension was defined as a systolic blood pressure (SBP) $\geq 140 \mathrm{mmHg}$, diastolic blood pressure $\geq 90 \mathrm{mmHg}$, or the use of antihypertensive medications. Diabetes was defined as a fasting blood glucose level $\geq 126 \mathrm{mg} / \mathrm{dL}$, nonfasting blood glucose level $\geq 200 \mathrm{mg} / \mathrm{dL}$, physician's reported diagnosis, or the use of hypoglycemic medications. CHD was defined as a history of MI, angina pectoris, or coronary revascularization.

\section{Assessment of Cognitive, Cardiac Function and Kidney Function}

The cognitive assessment of each participant was performed based on three cognitive tests in Visit 5. These tests included a delayed word recall test (DWRT), digit symbol substitution test (DSST), and word fluency test (WFT), which measured memory, executive function, and language function, respectively. ${ }^{24-28}$ The global cognition $z$-score was calculated as an average of the three individual z-scores and standardized using the global z-score mean and standard deviation (SD) of Visit 5.

Cardiac function was assessed using left ventricular ejection fraction (LVEF) based on echocardiography in Visit 5. According to the ARIC protocol, trained and certified sonographers performed echocardiographic examinations at the 5th visit, and quantitative measurements were done by the well-trained analysts at the central laboratory as recommended by the American Society of Echocardiography (ASE). ${ }^{29-32}$ In addition, the results were re-read by certified sonographers, which further ensured the accuracy of these measurements.

Kidney function was assessed by measuring creatinine in serum or plasma specimens collected at the 5th visit. And creatinine was measured by a modified kinetic Jaffé method. ${ }^{33-35}$

\section{Statistical Analysis}

Parametric variables were reported as mean \pm standard deviation and compared using variance analysis. Nonparametric variables were reported as median and interquartile range (25th-75th percentiles) and compared using the Mann-Whitney $U$-test. Categorical variables were reported as frequency and percentage and compared using the chi-square test.

To examine the association of SMI between the first and fourth visits with the incident frailty at the fifth visit, a logistic regression model was used. To further determine whether these relationships were independent of risk factors, the model was adjusted according to demographic variables (age, sex, race, education, annual household income, smoking, and alcohol consumption), physiological variables (BMI, SBP, and heart rate), laboratory findings (total cholesterol, low-density lipoprotein, high-density lipoprotein, triglycerides, and creatinine), and chronic medical conditions (hypertension, diabetes, and CHD). In addition, after adjusting for confounding factors, the logistic regression model analyzed the association between MI status and frailty in different subgroups, such as age, sex, race, hypertension, and diabetes and these interactions were examined. In order to calculate the indirect effect of MI status on frailty through cardiac (left ventricular ejection fraction [LVEF]), kidney (creatinine), and 
cognitive function (cognition score), path analysis by structural equation modeling was performed. The results of this analysis were reported using standardized regression coefficients $(\beta)$ to describe the direct and indirect effects on frailty. ${ }^{36}$

A two-tailed $P<0.05$ was considered significant for all tests. All statistical analyses were performed using SPSS version 26.0 (IBM Corp, Armonk, NY, USA) and R software 3.5.0 (Vienna, Austria).

\section{Results}

\section{Baseline Characteristics}

A total of 4953 participants with an average age of 52.2 \pm 5.1 years were included in this study. Among them, 2.7\% $(\mathrm{n}=135)$ and $2.9 \%(\mathrm{n}=146)$ developed SMI and CMI, respectively, between the first and fourth visits. Compared to non-MI, participants with SMI or CMI were more likely to be male; older; have chronic medical conditions, such as hypertension, diabetes, and CHD; have lower education level; and have more cardiovascular risk factors at the first visit. Baseline characteristics are described and compared in Table 1.

\section{Myocardial Infarction Status and Frailty}

After a median follow-up time of 14.7 (14.0-15.3) years, the average age of the participants at the 5th visit was 75.8 \pm 5.3 years. The total number of participants classified as pre-frail, frail, and robust were $46.7 \%(\mathrm{n}=2309), 6.7 \%$ $(n=336)$, and 46.6\% ( $\mathrm{n}=2308)$, respectively. Participants with SMI and CMI were significantly more likely to become frail after around 15 years than individuals without MI (15.6\% vs $6.2 \%, 16.4 \%$ vs $6.2 \%, P<0.001$ for all, respectively) (Figure 1).

In the logistic regression analysis, the unadjusted odds ratios (ORs) for SMI and frailty and CMI and frailty compared to participants without MI were $2.773(95 \%$ confidence interval [CI]: $1.716-4.483, P<0.001)$ and 3.111 (95\% CI: $1.990-4.862, P<0.001$ ), respectively. After adjusting for confounders, SMI and CMI were found to be independent predictors of frailty $(\mathrm{OR}=2.243$, 95\% CI: $1.307-3.850, P=0.003 ; \quad \mathrm{OR}=2.164,95 \%$ CI: 1.259-3.721, $P=0.005$ ) (Table 2).

\section{Subgroup Analysis}

The association between MI status and frailty was consistent among the subgroups of age, sex, race, diabetes, and hypertension (Figure 2). However, the risk of frailty associated with SMI was slightly higher, but not significant, for overweight than that for normalweight individuals ( $P$ for interaction $=0.065$ ), and for nonsmokers than that for smokers $(P$ for interaction $=0.097$ ).

\section{Path Analysis}

SMI and frailty were found to be significantly associated with LVEF and composite cognition score by path analysis $(P<0.05)$. In the structural equation modeling, the indirect effects of SMI on frailty by LVEF and composite cognition score were $27.9 \%(\beta=0.314,95 \%$ CI: $0.113-0.486)$ and $8.0 \%$ ( $\beta=0.091,95 \%$ CI: $0.021-0.178)$, respectively (Figure 3). The direct effect of SMI on frailty was $64.1 \%$ ( $\beta=0.722,95 \%$ CI: $0.160-1.284)$. Creatinine were not shown to be mediators of SMI and frailty. For patients with CMI (Table 3), the effects on frailty were mediated by LVEF (14.2\%), composite cognition score (11.4\%), and creatinine $(5.3 \%)$.

\section{Discussion}

This study found that patients with SMI and CMI were significantly more likely to be frail after around 15 years of MI. After adjusting for confounders, SMI and CMI were found to be independent predictors of frailty.

In our study, SMI was associated with a more than 2-fold increased risk of frailty while being independent of traditional cardiovascular risk factors. CMI was shown to have similar associations. However, previous studies have shown that patients with CMI are at an increased risk of adverse events, such as HF and SCD, than those with SMI. ${ }^{37,38}$ This could be due to the underdiagnosis of SMI, which can be easily screened by ECG. The prevalence of frailty in community-dwelling older adults $(\geq 60$ years of age) ranges from $3.9 \%$ to $51.4 \%$ and its presence is a risk factor for multiple adverse health outcomes that ultimately leads to hospitalization, falls, institutionalization, and death. ${ }^{39}$ Therefore, identifying individuals with SMI is of great importance and allows for earlier interventions against frailty and consequently, adverse health outcomes.

Even though other studies have shown that older female adults with hypertension and diabetes are at an increased risk of becoming frail, ${ }^{40-43}$ the association between different types of MI and frailty did not significantly differ by age, sex, and chronic medical conditions in our study. These results were similar to the study of the 
Table I Baseline (Visit-I, 1987-1989) Participant Characteristics Stratified by Myocardial Infarction Status

\begin{tabular}{|c|c|c|c|c|}
\hline \multirow[t]{2}{*}{ Characteristic } & Non-MI & Silent MI & Clinical MI & \multirow[t]{2}{*}{$\mathbf{P}$} \\
\hline & $(\mathrm{N}=4672)$ & $(\mathrm{N}=135)$ & $(N=146)$ & \\
\hline \multicolumn{5}{|l|}{ Demographic Variables } \\
\hline Age, years & $52.1 \pm 5.1$ & $53.5 \pm 4.9$ & $53.8 \pm 5.4$ & $<0.001$ \\
\hline Male sex & $1939(41.5)$ & $80(59.3)$ & $96(65.8)$ & $<0.001$ \\
\hline Race (Black) & $833(17.8)$ & $20(14.8)$ & $26(17.8)$ & 0.652 \\
\hline Physical activity score & $2.5 \pm 0.8$ & $2.4 \pm 0.7$ & $2.5 \pm 0.8$ & 0.117 \\
\hline Education & & & & $<0.001$ \\
\hline Less than high school & $569(12.2)$ & $26(19.3)$ & $35(24.0)$ & \\
\hline High school & 1525 (32.6) & $41(30.4)$ & $46(31.5)$ & \\
\hline College & $2578(55.2)$ & $68(50.4)$ & $65(44.5)$ & \\
\hline Smoking & & & & $<0.001$ \\
\hline Never & $2335(50.0)$ & $49(36.3)$ & $52(35.6)$ & \\
\hline Ever & $1568(33.6)$ & $47(34.8)$ & $59(40.4)$ & \\
\hline Current & $769(16.5)$ & $39(28.9)$ & $35(24.0)$ & \\
\hline Drinking & & & & 0.405 \\
\hline Never & $1090(23.3)$ & $24(17.8)$ & $33(22.6)$ & \\
\hline Ever & $638(13.7)$ & $31(23.0)$ & $27(18.5)$ & \\
\hline Current & $2944(63.0)$ & $80(59.3)$ & $86(58.9)$ & \\
\hline Income, US\$ & & & & 0.213 \\
\hline$<16,000$ & $518(11.1)$ & II (8.1) & $21(14.4)$ & \\
\hline $16,000-35,000$ & $1546(33.1)$ & $51(37.8)$ & $55(37.7)$ & \\
\hline$>35,000$ & $2608(55.8)$ & $73(54.1)$ & $70(47.9)$ & \\
\hline \multicolumn{5}{|c|}{ Physiological and Lab Variables } \\
\hline Body mass index, kg/m2 & $27.0 \pm 4.8$ & $28.4 \pm 4.8$ & $28.4 \pm 4.4$ & 0.002 \\
\hline $\mathrm{SBP}, \mathrm{mmHg}$ & $115.9 \pm 15.5$ & $120 \pm 16.8$ & $119.7 \pm 17$ & $<0.001$ \\
\hline $\mathrm{DBP}, \mathrm{mmHg}$ & $72.3 \pm 9.9$ & $74.5 \pm 11.1$ & $74.6 \pm 10.5$ & 0.002 \\
\hline Heart rate, $/ \mathrm{min}$ & $65.4 \pm 9.5$ & $64.6 \pm 9.2$ & $65 \pm 8.9$ & 0.615 \\
\hline Total cholesterol, $\mathrm{mmol} / \mathrm{l}$ & $5.4 \pm 1.0$ & $5.6 \pm 1.0$ & $5.8 \pm 1.2$ & $<0.001$ \\
\hline $\mathrm{HDL}, \mathrm{mmol} / \mathrm{l}$ & $1.4 \pm 0.4$ & $1.2 \pm 0.4$ & $1.2 \pm 0.4$ & $<0.001$ \\
\hline LDL, mmol/l & $3.5 \pm 1.0$ & $3.7 \pm 0.9$ & $3.8 \pm 1.1$ & $<0.001$ \\
\hline Triglycerides, mg/dl & $1.4 \pm 0.9$ & $1.5 \pm 0.8$ & $1.8 \pm 1.0$ & $<0.001$ \\
\hline Creatinine, $\mathrm{mg} / \mathrm{mL}$ & $1.1 \pm 0.2$ & $1.1 \pm 0.2$ & $1.1 \pm 0.2$ & $<0.001$ \\
\hline Blood glucose,mmol/l & $100.8 \pm 21.7$ & $105.9 \pm 34.7$ & $106.6 \pm 27.2$ & $<0.001$ \\
\hline \multicolumn{5}{|c|}{ Chronic Medical Conditions } \\
\hline Hypertension & $880(18.9)$ & $36(26.7)$ & $56(38.6)$ & $<0.001$ \\
\hline Diabetes mellitus & $178(3.8)$ & $6(4.5)$ & $16(11.0)$ & 0.001 \\
\hline Cancer & $227(4.9)$ & $9(6.7)$ & $7(4.8)$ & 0.662 \\
\hline COPD & $128(2.7)$ & $9(6.7)$ & $9(6.3)$ & 0.008 \\
\hline Coranary heart disease & $58(1.3)$ & $4(3.1)$ & $29(20.7)$ & $<0.001$ \\
\hline
\end{tabular}

Notes: Values are expressed as $n$ (\%), mean $\pm S D$, and median (25th, 75th).

Abbreviations: MI, myocardial infarction; SBP, systolic blood pressure; DBP, diastolic blood pressure; HDL, high density lipoprotein; LDL, Low density lipoprotein; COPD, chronic obstructive pulmonary disease. 


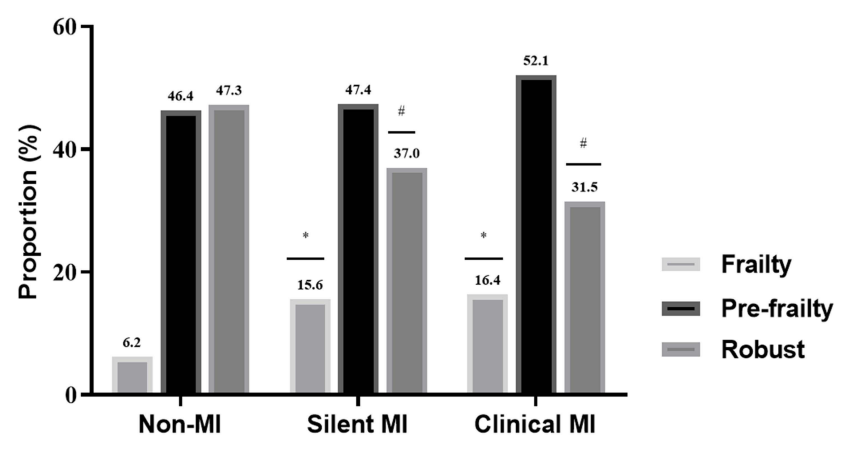

Figure I The prevalence of frailty in different MI status. ${ }^{*} \mathrm{P}<0.00 \mathrm{I}$. ${ }^{\#} \mathrm{P}<0.00 \mathrm{I}$. Abbreviation: MI, myocardial infarction.

association between SMI and risk of heart failure. Moreover, the same study reported that the risk of heart failure associated with SMI was higher in younger patients, but no such associations were found to be significant in our study.

Previous studies have confirmed that SMI leads to cardiac dysfunction, ${ }^{38}$ and abnormalities of cardiac structure and function are independently associated with frailty. ${ }^{44}$ Therefore, the increased risk of frailty in patients with SMI may be mediated by cardiac dysfunction, which was established in the path analysis performed in our study. According to our results, the indirect effects of SMI on frailty mediated by cardiac dysfunction (expressed by LVEF) accounted for about $28 \%$. Only HF with reduced ejection fraction is reflected by LVEF, but SMI could lead to HF with preserved ejection fraction. ${ }^{38}$ Therefore, the actual mediating effect value may be larger than the statistical value. Cognitive dysfunction, chronic kidney disease, and lung disease may also be mediators of SMI and frailty. ${ }^{45}$ Expectedly, cognition function accounted for $8 \%$ of the indirect effect of SMI on frailty in our study. This result emphasizes the importance of the heart-brain-frailty axis and could be an important intervention target. ${ }^{46}$ Chronic kidney disease, expressed as creatinine $(5.3 \%)$, was shown to be a mediator of frailty in patients with CMI, but this was not observed in patients with SMI. Patients with CMI may have had a larger infarct size, whereas those with SMI are likely to remain subclinical due to the presence of small infarct size. Therefore, patients with CMI could be at an increased risk of developing kidney disease compared with those with SMI.

\section{Limitations}

This study has several limitations. Only participants diagnosed with MI between the first and fourth visits were included to analyze the relationship between MI and the risk of frailty over a span of 15 years. The baseline frailty status of the study population could not be obtained, thus a causal relationship could not be proven. In addition, frailty was diagnosed based on the frailty phenotype, and it is unclear whether this would be relevant when using other proposed criteria. Since the assessment of lung function was only conducted in the first and second visits of the study, it were not included in the path analysis. Moreover, older adult frail individuals are at an increased risk of early-onset death and loss to followup, leading to selection bias and uncertainty of the research conclusion. However, this is almost an inevitable problem in most studies. Although confounders were adjusted for in this study, there may still be potential variables that were not included. Finally, CMI may be derived from recurrent MI of the population with SMI, thus, the true SMI events may be underestimated.

Table 2 Adjusted ORs $(95 \% \mathrm{Cl})$ for the Association of Silent MI and Clinical MI with Incident Frailty

\begin{tabular}{|c|c|c|c|c|c|c|c|c|}
\hline \multirow[t]{2}{*}{ Diagnose } & \multicolumn{2}{|l|}{ Unadjusted } & \multicolumn{2}{|l|}{ Model I } & \multicolumn{2}{|l|}{ Model 2} & \multicolumn{2}{|l|}{ Model 3} \\
\hline & OR $(95 \% \mathrm{Cl})$ & $\mathbf{P}$ & OR (95\% Cl) & $\mathbf{P}$ & OR $(95 \% \mathrm{CI})$ & $\mathbf{P}$ & OR $(95 \% \mathrm{CI})$ & $\mathbf{P}$ \\
\hline MI status & & $<0.001$ & & $<0.001$ & & $<0.001$ & & $<0.001$ \\
\hline Non-MI & Ref. & - & Ref. & - & Ref. & - & Ref. & - \\
\hline Silent MI & $2.773(1.716-4.483)$ & $<0.001$ & $2.788(1.708-4.550)$ & $<0.001$ & $2.423(1.432-4.099)$ & 0.001 & $2.243(1.307-3.850)$ & 0.003 \\
\hline Clinical MI & 3.111 (I.990-4.862) & $<0.001$ & $3.032(1.912-4.808)$ & $<0.001$ & $2.062(1.210-3.513)$ & 0.008 & $2.164(1.259-3.721)$ & 0.005 \\
\hline
\end{tabular}

Notes: Model I: adjusted by age, sex, center-race. Model 2: adjusted by model I plus education (<high school, high school, or >high school), annual household income ( $<16,000,16,000$ to 35,000, $>35,000$ ), and body mass index, smoking (never, former, current), drinking (never, former, current), systolic blood pressure, heart rate. Model 3: adjusted by model 2 plus physical activity score, total cholesterol, low density lipoprotein, high density lipoprotein, triglycerides, creatinine, hypertension, diabetes, and coronary heart disease. Unexplained variables are regarded as continuous variables.

Abbreviations: $\mathrm{MI}$, myocardial infarction; OR, odds ratio; $\mathrm{Cl}$, confidence interval. 


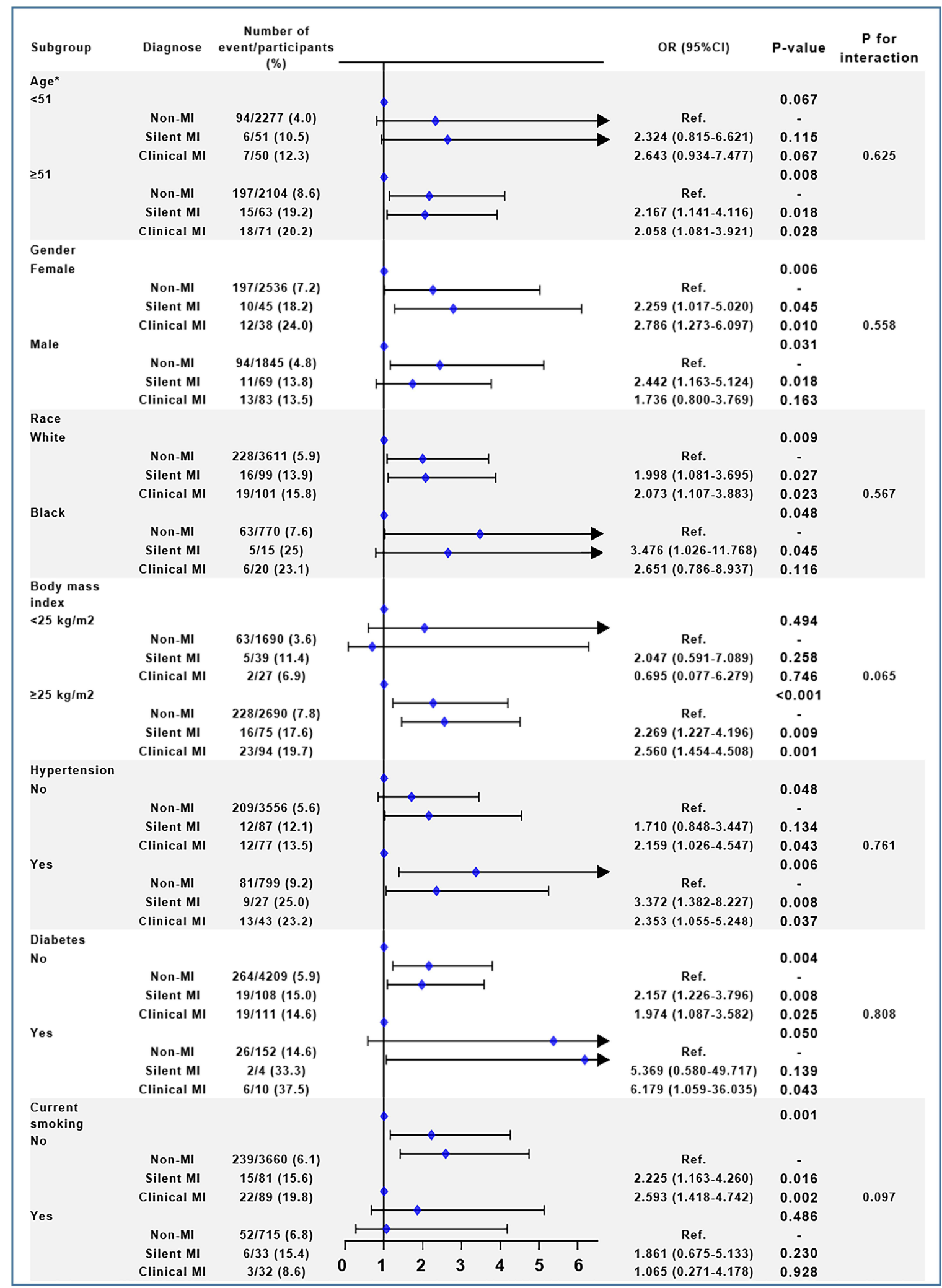

Figure 2 Adjusted ORs ( $95 \% \mathrm{Cls})$ for the association of $\mathrm{Ml}$ status with incident frailty in different subgroups. Model was adjusted by age, sex, center-race, education (<high school, high school, or $>$ high school), annual household income $(<16,000,16,000$ to $35,000,>35,000)$, and body mass index, smoking (never, former, current), drinking (never, former, current), systolic blood pressure, heart rate, physical activity score, total cholesterol, low density lipoprotein, high density lipoprotein, triglycerides, creatinine, hypertension, diabetes, and coronary heart disease.

Abbreviations: $\mathrm{Ml}$, myocardial infarction; $\mathrm{OR}$, odds ratio; $\mathrm{Cl}$, confidence interval. 


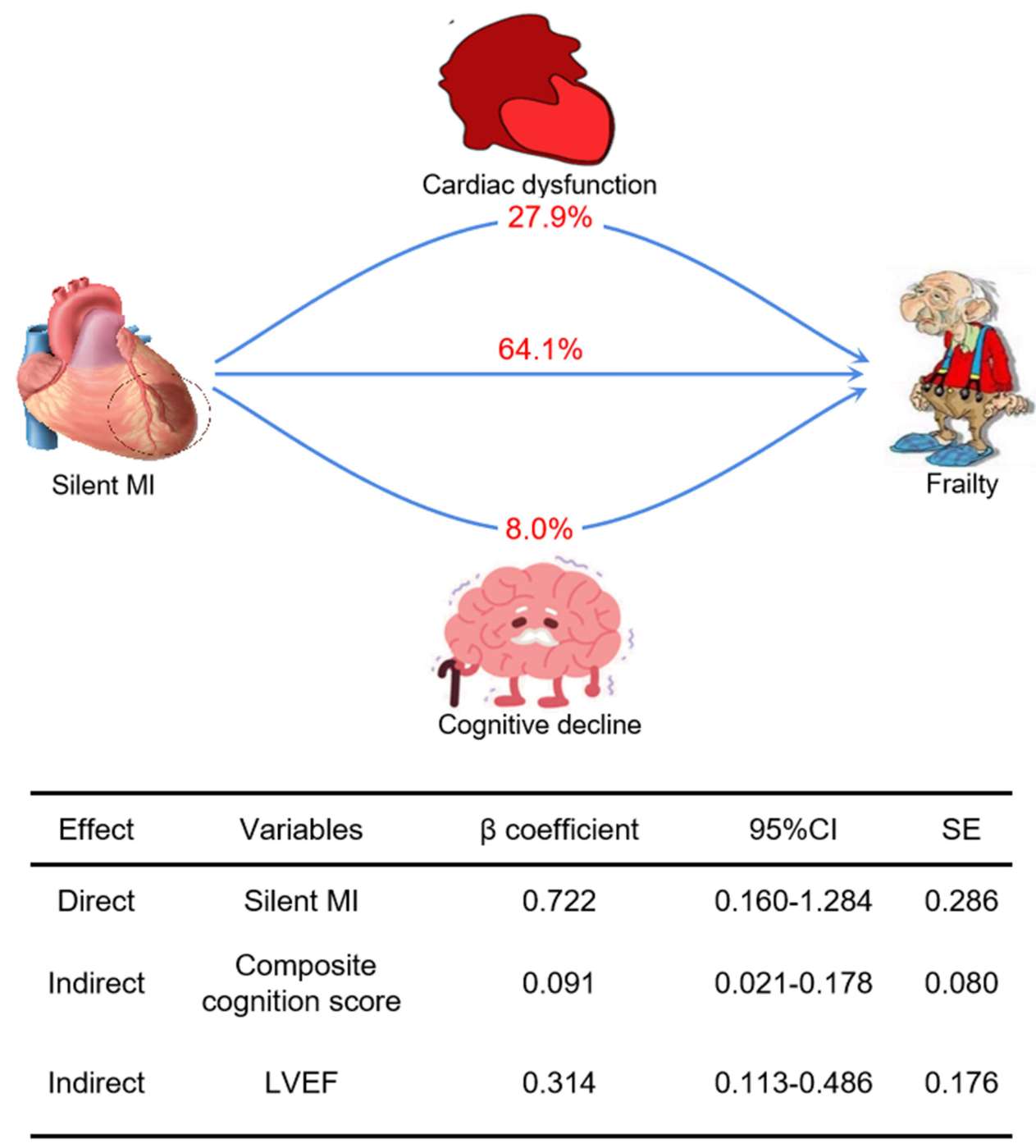

Figure 3 Direct and indirect effects of silent MI on frailty. $\beta$ coefficient was calculated by standard regression equation. Composite cognition score was calculated by mean of Digit Symbol Substitution Test $Z$ score, Word Fluency Test $Z$ score, and Delayed Word Recall $Z$ score.

Abbreviations: $\mathrm{MI}$, myocardial infarction; LVEF, left ventricular ejection fraction; Cl, confidence interval; SE, standard error.

\section{Conclusions}

This study found that individuals with MI were at an increased risk of becoming frail. This association is more important in patients with SMI as they are more likely to be underdiagnosed. In addition, the mechanism between SMI and frailty can be explained by the mediation of cardiac and cognitive function impairment. Identifying individuals with SMI may allow for earlier interventions to prevent the progress of frailty. Standardized preventive treatment needs to be

Table 3 Direct and Indirect Effects of Clinical MI on Frailty

\begin{tabular}{|l|c|c|c|c|}
\hline Effect & Variables & B Coefficient & $\mathbf{9 5 \%}$ Cl & SE \\
\hline Direct & Clinical MI & 0.394 & $0.160-0.628$ & 0.120 \\
Indirect & Composite cognition score & 0.079 & $0.035-0.121$ & 0.015 \\
Indirect & LVEF & 0.101 & $0.056-0.397$ & 0.031 \\
Indirect & Serum creatinine & 0.034 & $0.010-0.098$ & 0.009 \\
\hline
\end{tabular}

Notes: $\beta$ coefficient was calculated by standard regression equation. Composite cognition score was calculated by mean of Digit Symbol Substitution Test $Z$ score, Word Fluency Test $Z$ score, and Delayed Word Recall $Z$ score.

Abbreviations: MI, myocardial infarction; LVEF, left ventricular ejection fraction. 
established to reduce the risk of frailty among patients with SMI.

\section{Abbreviations}

ARIC, Atherosclerosis Risk in Communities; BMI, body mass index; CAD, coronary artery disease; CHD, coronary heart disease; CI, confidence interval; CMI, clinical myocardial infarction; COPD, chronic obstructive pulmonary disease; CVD, cardiovascular disease; DBP, diastolic blood pressure; ECG, electrocardiogram; HF, heart failure; HDL, high density lipoprotein; LDL, Low density lipoprotein; LVEF, left ventricular ejection fraction; MI, myocardial infarction; OR, odds ratio; SMI, Silent myocardial infarction; SCD, sudden cardiac death; SBP, systolic blood pressure.

\section{Ethics Approval and Informed Consent}

The study was conducted in agreement with the Declaration of Helsinki and was approved by the Institutional Review Committee.

\section{Acknowledgments}

The authors thank the staff and participants of the ARIC study, and BioLINCC for their important contributions.

\section{Author Contributions}

All authors made substantial contributions to conception and design, acquisition of data, or analysis and interpretation of data; took part in drafting the article or revising it critically for important intellectual content; agreed to submit to the current journal; gave final approval of the version to be published; and agree to be accountable for all aspects of the work.

\section{Funding}

This work was supported financially by grants from Sichuan Science and Technology Program (No. 2020YFS0154, 2020YFSY0014), $1 \cdot 3 \cdot 5$ Project for Disciplines of Excellence-Clinical Research Incubation Project, Sichuan University West China Hospital (No. 2018HXFH001, and 2018HXFH027, 2020HXFH050), Sichuan University West China Hospital (No. CGZH19008), Sichuan University West China Nursing Discipline Development Special Fund Project (No. HXHL20046, HXHL19023), Chengdu Science and Technology Bureau (No. 2019-YF05-00322-SN), National
Clinical Research Center for Geriatrics, West China Hospital, Sichuan University (No. Z20191009).

\section{Disclosure}

The authors declare that there is no conflict of interest.

\section{References}

1. Singh M, Stewart R, White H. Importance of frailty in patients with cardiovascular disease. Eur Heart J. 2014;35(26):1726-1731. doi:10.1093/eurheartj/ehu197

2. Matsuoka M, Inoue T, Shinjo T, et al. Cardiovascular risk profile and frailty in Japanese outpatients: the Nambu Cohort Study. Hypertens Res. 2020;43(8):817-823. doi:10.1038/s41440-020-0427-z

3. Collard RM, Boter H, Schoevers RA, Oude Voshaar RC. Prevalence of frailty in community-dwelling older persons: a systematic review. $J$ Am Geriatr Soc. 2012;60(8):1487-1492. doi:10.1111/j.15325415.2012.04054.x

4. Gale CR, Cooper C, Sayer AA. Prevalence of frailty and disability: findings from the English Longitudinal Study of Ageing. Age Ageing. 2015;44(1):162-165. doi:10.1093/ageing/afu148

5. Stewart R. Cardiovascular disease and frailty: what are the mechanistic links? Clin Chem. 2019;65(1):80-86. doi:10.1373/ clinchem.2018.287318

6. Bebb O, Smith FG, Clegg A, Hall M, Gale CP. Frailty and acute coronary syndrome: a structured literature review. Eur Heart $J$ Acute Cardiovasc Care. 2018;7(2):166-175. doi:10.1177/ 2048872617700873

7. Patel MR, Calhoon JH, Dehmer GJ, et al. ACC/AATS/AHA/ASE/ ASNC/SCAI/SCCT/STS 2016 appropriate use criteria for coronary revascularization in patients with acute coronary syndromes: a report of the American College of Cardiology Appropriate Use Criteria Task Force, American Association for Thoracic Surgery, American Heart Association, American Society of Echocardiography, American Society of Nuclear Cardiology, Society for Cardiovascular Angiography and Interventions, Society of Cardiovascular Computed Tomography, and the Society of Thoracic Surgeons. $J$ Am Coll Cardiol. 2017;69(5):570-591. doi:10.1016/j. jacc.2016.10.034

8. Collet JP, Thiele H, Barbato E, et al. 2020 ESC guidelines for the management of acute coronary syndromes in patients presenting without persistent ST-segment elevation. Eur Heart J. 2020;41:3495-3497. doi:10.1093/eurheartj/ehaa624

9. Li F, Li D, Yu J, et al. Barthel index as a predictor of mortality in patients with acute coronary syndrome: better activities of daily living, better prognosis. Clin Interv Aging. 2020;15:1951-1961. doi:10.2147/CIA.S270101

10. Jia Y, Li H, Li D, et al. Prognostic value of braden scale in patients with acute myocardial infarction: from the retrospective multicenter study for early evaluation of acute chest pain. J Cardiovasc Nurs. 2020;35(6):E53-e61. doi:10.1097/JCN.0000000000000735

11. Myers V, Drory Y, Gerber Y. Clinical relevance of frailty trajectory post myocardial infarction. Eur J Prev Cardiol. 2014;21(6):758-766. doi: $10.1177 / 2047487312462828$

12. Li D, Cheng Y, Yu J, et al. Early risk stratification of acute myocardial infarction using a simple physiological prognostic scoring system: insights from the REACP study. Eur $J$ Cardiovasc Nurs. 2021;20(2):167-174. doi:10.1177/1474515120952214

13. Jia Y, Li D, Cao Y, et al. Inflammation-based glasgow prognostic score in patients with acute ST-segment elevation myocardial infarction: a prospective cohort study. Medicine. 2018;97(50):e13615. doi:10.1097/MD.0000000000013615 
14. Schelbert EB, Cao JJ, Sigurdsson S, et al. Prevalence and prognosis of unrecognized myocardial infarction determined by cardiac magnetic resonance in older adults. JAMA. 2012;308(9):890-896. doi:10.1001/2012.jama.11089

15. Amier RP, Smulders MW, van der Flier WM, et al. Long-term prognostic implications of previous silent myocardial infarction in patients presenting with acute myocardial infarction. JACC Cardiovasc Imaging. 2018;11(12):1773-1781. doi:10.1016/j. jcmg.2018.02.009

16. Zhang ZM, Rautaharju PM, Prineas RJ, et al. Race and sex differences in the incidence and prognostic significance of silent myocardial infarction in the Atherosclerosis Risk in Communities (ARIC) study. Circulation. 2016;133(22):2141-2148. doi:10.1161/ CIRCULATIONAHA.115.021177

17. Turkbey EB, Nacif MS, Guo M, et al. Prevalence and correlates of myocardial scar in a US cohort. JAMA. 2015;314(18):1945-1954. doi:10.1001/jama.2015.14849

18. Kehl DW, Farzaneh-Far R, Na B, Whooley MA. Prognostic value of electrocardiographic detection of unrecognized myocardial infarction in persons with stable coronary artery disease: data from the Heart and Soul Study. Clin Res Cardiol. 2011;100(4):359-366. doi:10.1007/s00392-010-0255-2

19. Vahatalo JH, Huikuri HV, Holmstrom LTA, et al. Association of silent myocardial infarction and sudden cardiac death. JAMA Cardiol. 2019;4(8):796-802. doi:10.1001/jamacardio.2019.2210

20. Nadruz W, Kitzman D, Windham BG, et al. Cardiovascular dysfunction and frailty among older adults in the community: the ARIC study. J Gerontol Series A. 2017;72(7):958-964.

21. Fried LP, Tangen CM, Walston J, et al. Frailty in older adults: evidence for a phenotype. J Gerontol A Biol Sci Med Sci. 2001;56 (3):M146-156. doi:10.1093/gerona/56.3.M146

22. Hertogh EM, Monninkhof EM, Schouten EG, Peeters PH, Schuit AJ. Validity of the modified Baecke questionnaire: comparison with energy expenditure according to the doubly labeled water method. Int $J$ Behav Nutr Phys Act. 2008;5:30. doi:10.1186/1479-5868-5-30

23. Cobb LK, Godino JG, Selvin E, Kucharska-Newton A, Coresh J, Koton S. Spousal influence on physical activity in middle-aged and older adults: the ARIC study. Am J Epidemiol. 2016;183(5):444-451. doi:10.1093/aje/kwv104

24. Kim SM, Zhao D, Schneider ALC, et al. Association of parathyroid hormone with 20-year cognitive decline: the ARIC study. Neurology. 2017;89(9):918-926. doi:10.1212/WNL.0000000000004290

25. Li D, Misialek JR, Jing M, et al. Plasma phospholipid very-longchain SFAs in midlife and 20-year cognitive change in the Atherosclerosis Risk in Communities (ARIC): a cohort study. Am $J$ Clin Nutr. 2020;111(6):1252-1258. doi:10.1093/ajcn/nqaa048

26. Knopman DS, Ryberg S. A verbal memory test with high predictive accuracy for dementia of the Alzheimer type. Arch Neurol. 1989;46 (2):141-145. doi:10.1001/archneur.1989.00520380041011

27. Jaeger J. Digit symbol substitution test: the case for sensitivity over specificity in neuropsychological testing. J Clin Psychopharmacol. 2018;38(5):513-519. doi:10.1097/JCP.0000000000000941

28. Pendleton MG, Heaton RK, Lehman RA, Hulihan D. Diagnostic utility of the Thurstone Word Fluency Test in neuropsychological evaluations. J Clin Neuropsychol. 1982;4(4):307-317. doi:10.1080/ 01688638208401139

29. Shah AM, Cheng S, Skali H, et al. Rationale and design of a multicenter echocardiographic study to assess the relationship between cardiac structure and function and heart failure risk in a biracial cohort of community-dwelling elderly persons: the Atherosclerosis Risk in Communities study. Circ Cardiovasc Imaging. 2014;7(1):173-181. doi:10.1161/CIRCIMAGING.113.00 0736
30. Lang RM, Bierig M, Devereux RB, et al. Recommendations for chamber quantification: a report from the American Society of Echocardiography's guidelines and standards committee and the chamber quantification writing group, developed in conjunction with the European Association of Echocardiography, a branch of the European Society of Cardiology. J Am Soc Echocardiograph. 2005;18(12):1440-1463. doi:10.1016/j.echo.2005.10.005

31. Nagueh SF, Appleton CP, Gillebert TC, et al. Recommendations for the evaluation of left ventricular diastolic function by echocardiography. Eur $J$ Echocardiograp. 2009;10(2):165-193. doi:10.1093/ejechocard/jep007

32. Rudski LG, Lai WW, Afilalo J, et al. Guidelines for the echocardiographic assessment of the right heart in adults: a report from the American Society of Echocardiography endorsed by the European Association of Echocardiography, a registered branch of the European Society of Cardiology, and the Canadian Society of Echocardiography. J Am Soc Echocardiograph. 2010;23(7):685713; quiz 786-688. doi:10.1016/j.echo.2010.05.010

33. Eckfeldt JH, Chambless LE, Shen YL. Short-term, within-person variability in clinical chemistry test results. Experience from the Atherosclerosis Risk in Communities Study. Arch Pathol Lab Med. 1994;118(5):496-500.

34. Coresh J, Astor BC, McQuillan G, et al. Calibration and random variation of the serum creatinine assay as critical elements of using equations to estimate glomerular filtration rate. Am J Kidney Dis. 2002;39(5):920-929. doi:10.1053/ajkd.2002.32765

35. Parrinello CM, Grams ME, Couper D, et al. Recalibration of blood analytes over 25 years in the atherosclerosis risk in communities study: impact of recalibration on chronic kidney disease prevalence and incidence. Clin Chem. 2015;61(7):938-947. doi:10.1373/ clinchem.2015.238873

36. Stein CM, Morris NJ, Hall NB, Nock NL. Structural equation modeling. Methods Mol Biol. 2017;1666:557-580.

37. Cheng YJ, Jia YH, Yao FJ, et al. Association between silent myocardial infarction and long-term risk of sudden cardiac death. $\mathrm{J} \mathrm{Am}$ Heart Assoc. 2021;10(1):e017044. doi:10.1161/JAHA.120.017044

38. Qureshi WT, Zhang ZM, Chang PP, et al. Silent myocardial infarction and long-term risk of heart failure: the ARIC study. $\mathrm{J} \mathrm{Am} \mathrm{Coll}$ Cardiol. 2018;71(1):1-8. doi:10.1016/j.jacc.2017.10.071

39. Siriwardhana DD, Hardoon S, Rait G, Weerasinghe MC, Walters KR. Prevalence of frailty and prefrailty among community-dwelling older adults in low-income and middle-income countries: a systematic review and meta-analysis. BMJ Open. 2018;8(3):e018195. doi:10.1136/bmjopen-2017-018195

40. Aprahamian I, Sassaki E, Dos Santos MF, et al. Hypertension and frailty in older adults. J Clin Hypertens. 2018;20(1):186-192. doi:10.1111/jch.13135

41. Yanase T, Yanagita I, Muta K, Nawata H. Frailty in elderly diabetes patients. Endocr J. 2018;65(1):1-11. doi:10.1507/endocrj.EJ17-0390

42. Washington SL, Porten SP, Quanstrom K, et al. The association between race and frailty in older adults presenting to a nononcologic urology practice. Urology. 2019;127:19-23. doi:10.1016/j.urology.2019.02.024

43. Hubbard RE. Sex differences in frailty. Interdisciplin Topics Gerontol Geriatr. 2015;41:41-53.

44. Soysal P, Arik F, Smith L, Jackson SE, Isik AT. Inflammation, frailty and cardiovascular disease. Adv Exp Med Biol. 2020;1216:55-64.

45. Shen Z, Ruan Q, Yu Z, Sun Z. Chronic kidney disease-related physical frailty and cognitive impairment: a systemic review. Geriatr Gerontol Int. 2017;17(4):529-544. doi:10.1111/ggi.12758

46. Butts B, Gary R. Coexisting frailty, cognitive impairment, and heart failure: implications for clinical care. J Clin Outcomes Manag. 2015;22(1):38-46. 


\section{Publish your work in this journal}

Clinical Interventions in Aging is an international, peer-reviewed journal focusing on evidence-based reports on the value or lack thereof of treatments intended to prevent or delay the onset of maladaptive correlates of aging in human beings. This journal is indexed on PubMed Central, MedLine, CAS, Scopus and the Elsevier
Bibliographic databases. The manuscript management system is completely online and includes a very quick and fair peer-review system, which is all easy to use. Visit http://www.dovepress.com/ testimonials.php to read real quotes from published authors.

Submit your manuscript here: https://www.dovepress.com/clinical-interventions-in-aging-journal 